\title{
The Importance of Biological Particles to the Ice Nucleating Particle Concentration in a Coastal Tropical Site
}

\author{
Luis A. Ladino ${ }^{1, *}$, Graciela B. Raga ${ }^{1}$, Harry Alvarez-Ospina ${ }^{2}$, Manuel A. \\ Andino-Enriquez ${ }^{3}$, Irma Rosas ${ }^{1}$, Leticia Martinez ${ }^{1}$, Eva Salinas ${ }^{1}$, Javier \\ Miranda $^{4}$, Zyanya Ramirez-Diaz ${ }^{1}$, Bernardo Figueroa ${ }^{5}$, Cedric Chou ${ }^{6}$, Allan K. \\ Bertram $^{6}$, Erika T. Quintana7, Luis A. Maldonado ${ }^{8}$, Agustín Garcia-Reynoso", \\ Meng $\mathrm{Si}^{6}$, and Victoria $\mathrm{E}$. Irish ${ }^{6}$
}

${ }^{1}$ Centro de Ciencias de la Atmosfera, Universidad Nacional autónoma de México, Mexico City, Mexico

${ }^{2}$ Facultad de Ciencias, Universidad Nacional Autónoma de México, Mexico City, Mexico

${ }^{3}$ School of Chemical Sciences and Engineering, Universidad Yachay Tech, Ecuador

${ }^{4}$ Instituto de Física, Universidad Nacional Autónoma de México, Mexico City, Mexico

${ }^{5}$ Laboratorio de Ingeniería y Procesos Costeros, Instituto de Ingeniería, Universidad Nacional Autónoma de México, Sisal, Yucatán, Mexico

${ }^{6}$ Chemistry Department, University of British Columbia, Vancouver, Canada.

${ }^{7}$ Escuela Nacional de Ciencias Biológicas, Instituto Politécnico Nacional, Mexico City, México

${ }^{8}$ Facultad de Química, Universidad Nacional autónoma de México, Mexico City, Mexico

${ }^{*}$ Correspondence to: Luis A. Ladino (luis.ladino@atmosfera.unam.mx)

\section{Supplemental Material}

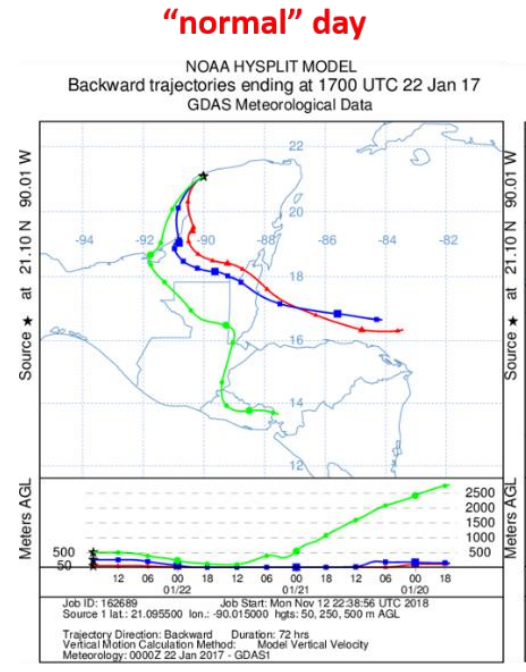

South, east and west winds
Cold front A

NOAA HYSPLIT MODEL

UTC 24 Jan 17 GDAS Meteorological Data

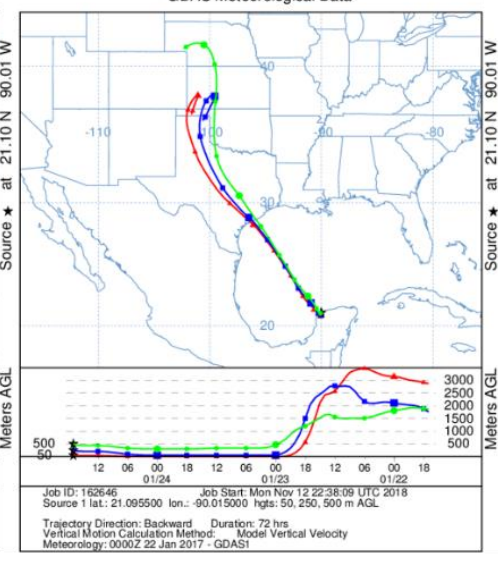

Northwest winds
Cold front $B$

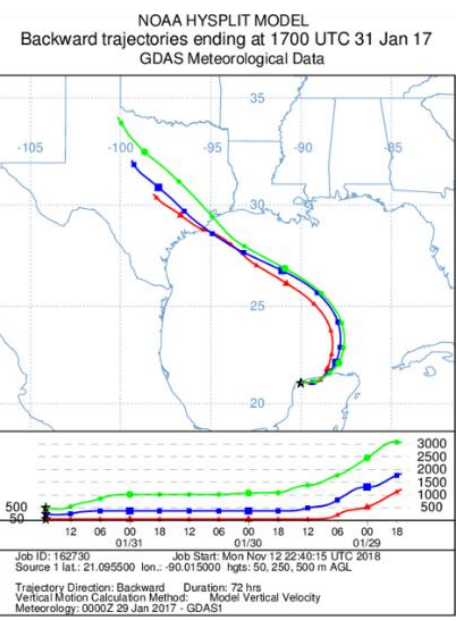

North winds

Figure S1. Examples of the Hysplit back-trajectories for $72 \mathrm{~h}$ on a left) "normal" day, middle) cold front $A$, and right) cold front $B$. 

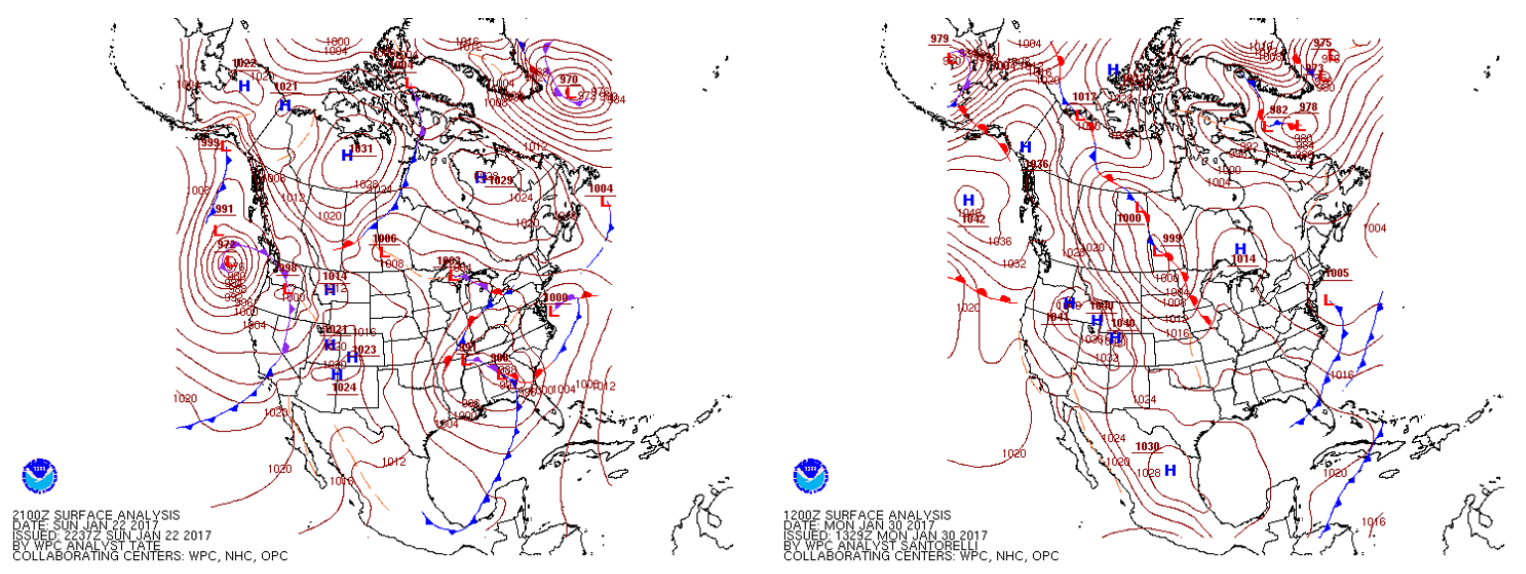

Figure S2. NOAA surface maps showing the arrival and passage of left) cold front $A$, and right) cold front $B$.

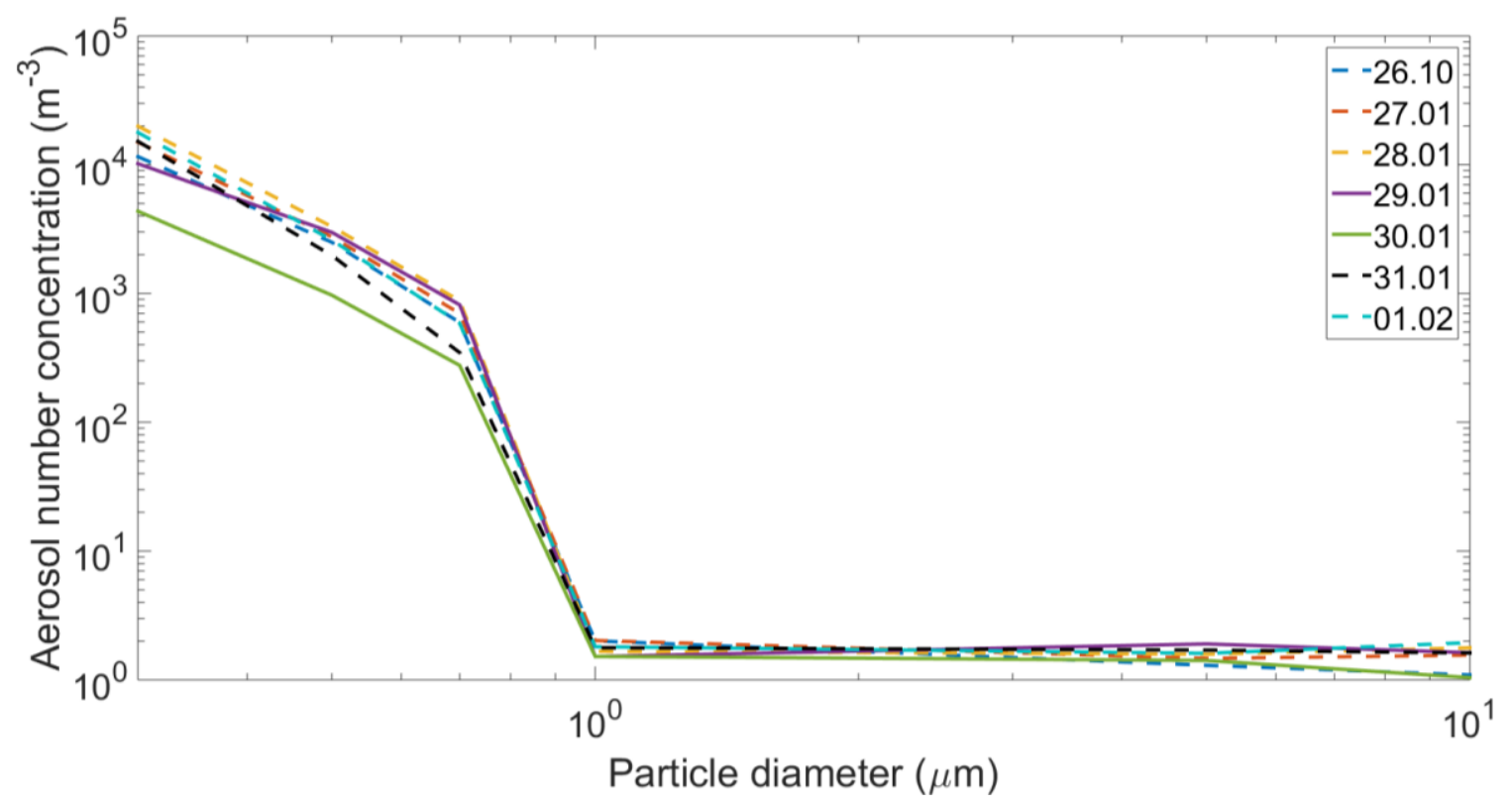

Figure S3. Examples of the aerosol number size distributions before (January 26 to 28), during (January 29 and 30) and after (January 31 and February 01 ) the passage of a cold air mass. 

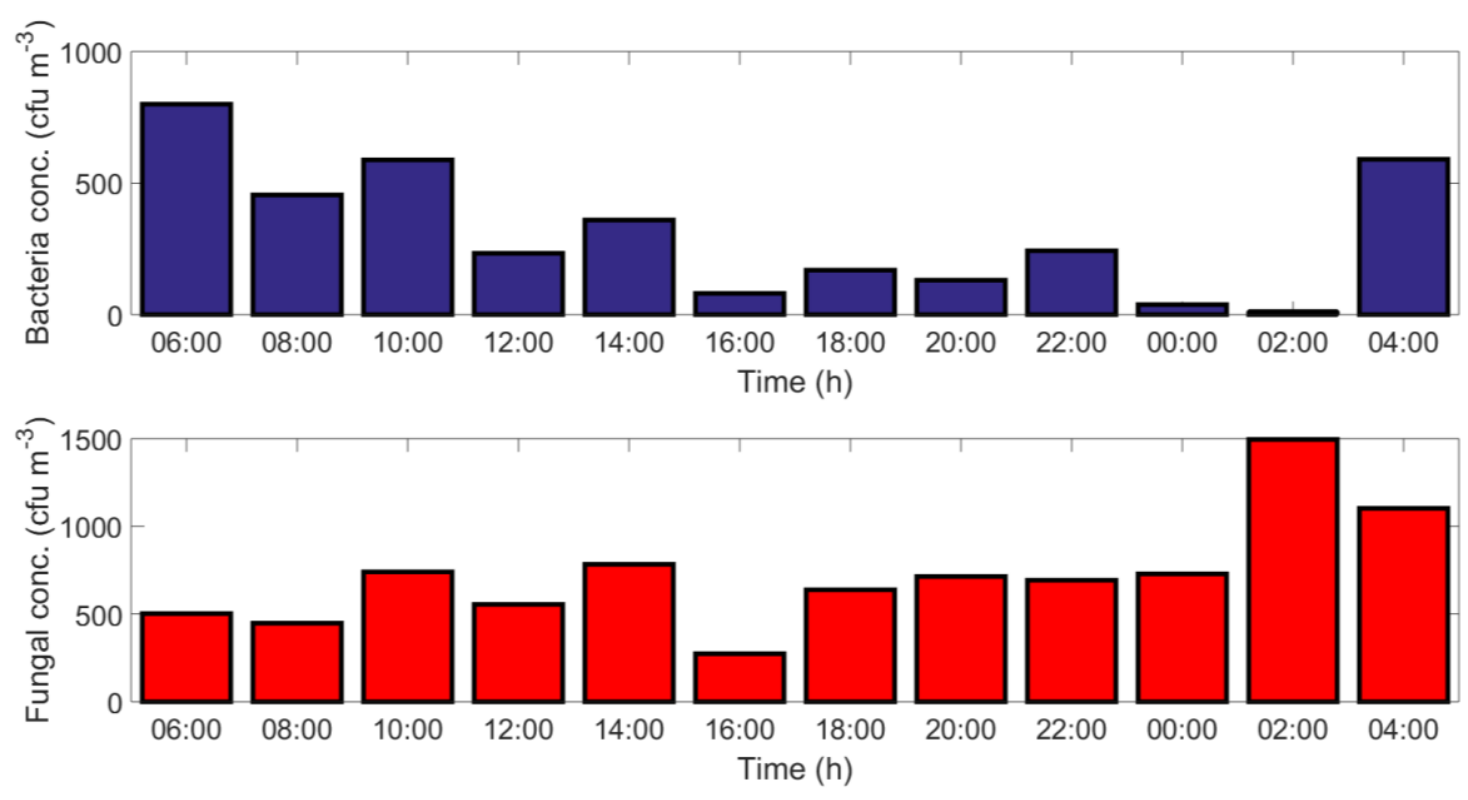

Figure S4. Daily profile of the Top) bacteria, and Bottom) fungal concentration (cfu m${ }^{-3}$ ) between January 22 and 23, 2017.
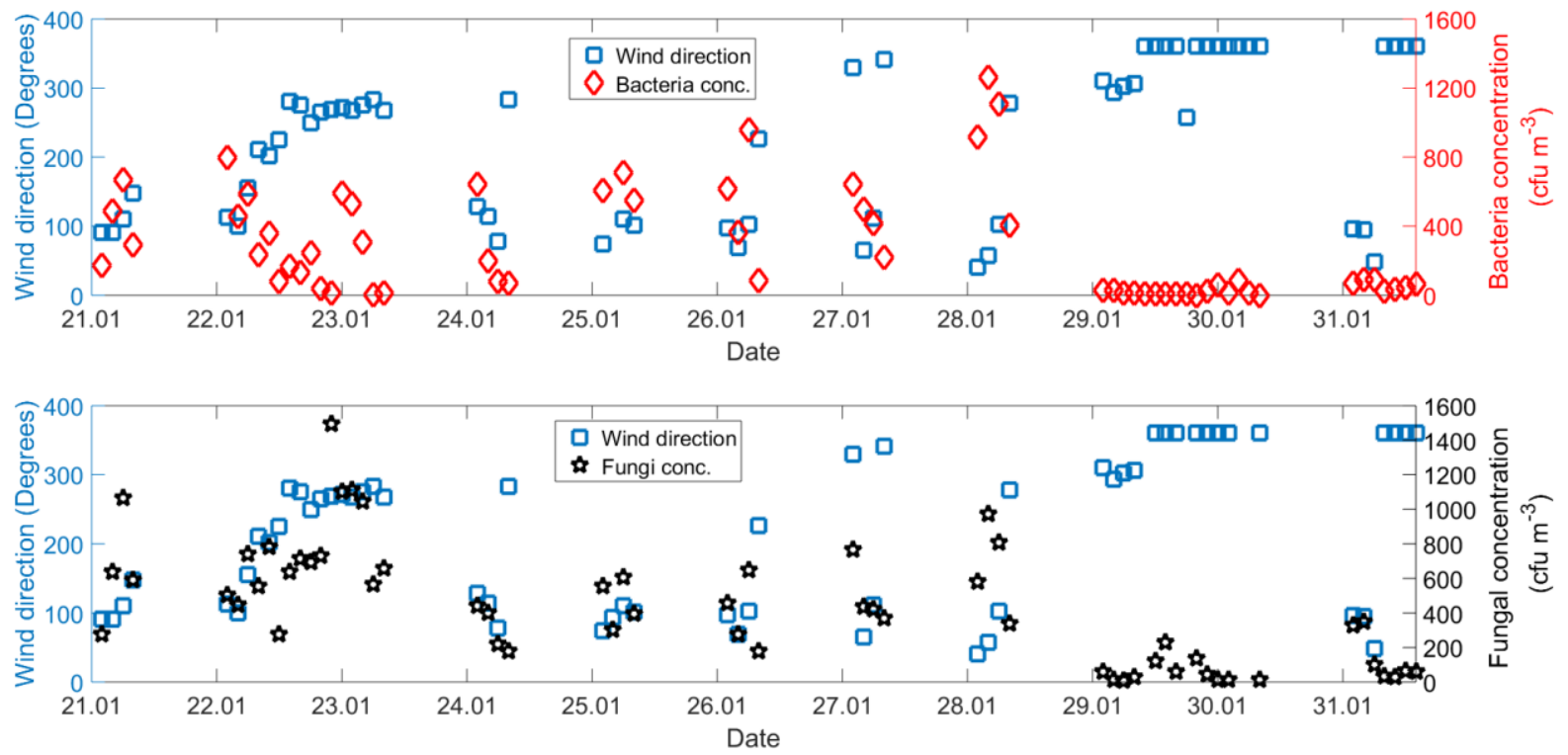

Figure S5. Time series of the Top) wind direction vs. bacteria concentration, and Bottom) wind direction vs. fungal concentration between January 21 and 31. Each X-axis tick corresponds to 6:00 a.m. local time. 


\section{B. Fungal propagule}

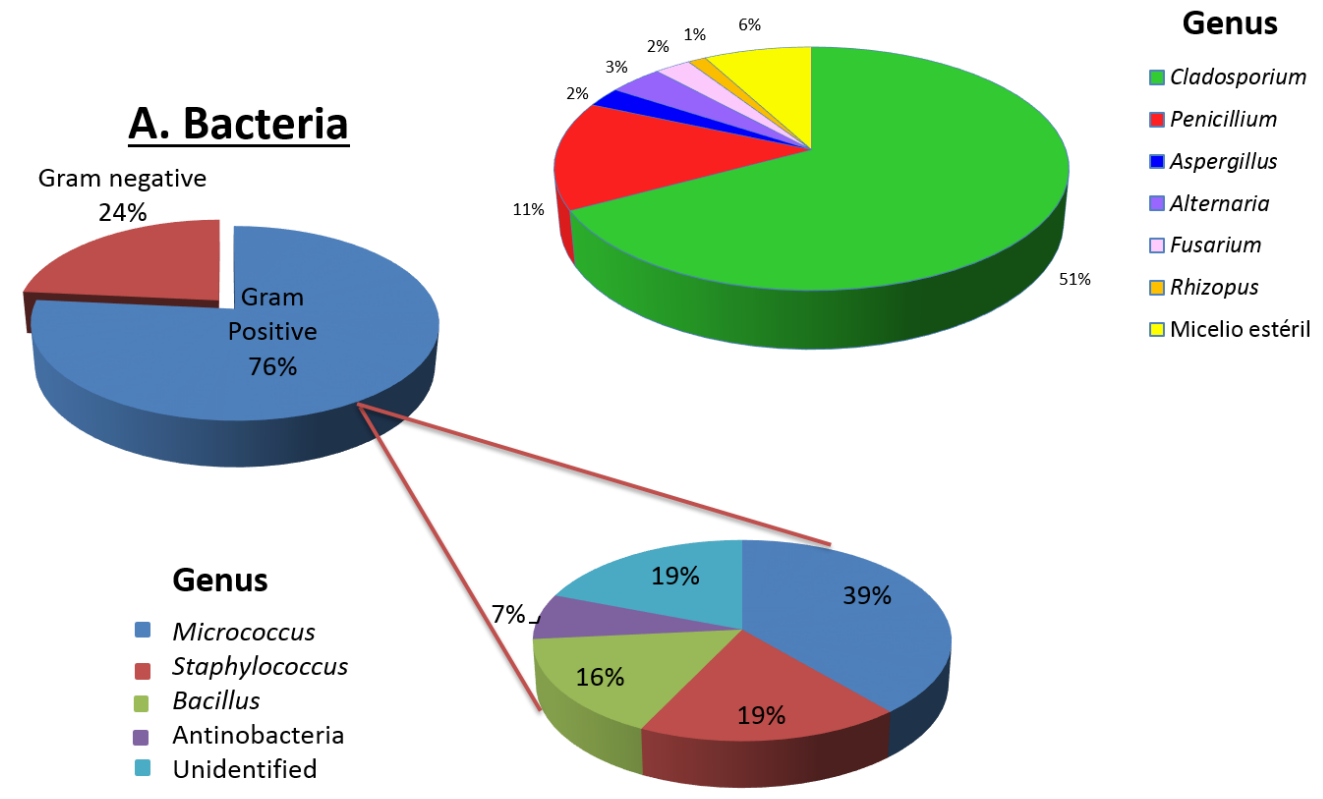

Figure S6. A) Gram bacteria staining analysis for the whole sampling period and, B) Identification of the Fungal propagules for the whole sampling period. 